\title{
Understanding the interplay of lies, violence, and religious values in folktales
}

\author{
Quan-Hoang Vuong ${ }^{1}$ \\ Centre for Interdisciplinary Social Research, Phenikaa University \\ Yen Nghia, Ha Dong district, Hanoi 100803, Vietnam \\ hoang.vuongquan@phenikaa-uni.edu.vn \\ Viet-Phuong La \\ Centre for Interdisciplinary Social Research, Phenikaa University \\ Yen Nghia, Ha Dong district, Hanoi 100803, Vietnam \\ phuong.laviet@phenikaa-uni.edu.vn \\ Hong-Kong T. Nguyen \\ Graduate School of Asia Pacific Studies, Ritsumeikan Asia Pacific University \\ Beppu, Oita Prefecture, 874-8577, Japan \\ tohong19@apu.ac.jp \\ Working Paper No. PKU-1905 \\ Hanoi, June 7, 2019
}

Abstract

This research employs the Bayesian network modeling approach, and the Markov chain Monte Carlo technique, to learn about the role of lies and violence in teachings of major religions, using a unique dataset extracted from long-standing Vietnamese folktales. The results indicate that, although lying and violent acts augur negative consequences for those who commit them, their associations with core religious values diverge in the outcome for the folktale characters. Lying that serves a religious mission of either Confucianism or Taoism (but not Buddhism) brings a positive outcome to a character. A violent act committed to serving Buddhist mission results in a happy ending for the committer.

\footnotetext{
1 Corresponding author.
} 


\title{
Understanding the interplay of lies, violence, and religious values in folktales
}

\author{
Quan-Hoang Vuong, Viet-Phuong La, and Hong-Kong T. Nguyen
}

The complexity of human decision-making means certain contradictions are inevitable, if not necessary, for reaching the desired outcome. This issue is compounded in the face of religion, which is seen as a strong binding factor of moral communities (1) and yet is also the underlying reason for most violent conflicts in human history $(2,3)$. How are we to make sense of the positive outcome resulted from lying and violent acts even though religious and ethical values are embedded throughout?

Using a unique dataset strongly representative of long-standing Vietnamese folktales, this study offers the first-ever Bayesian network model to examine the interplay of lies and violence in religiously-charged narrative settings. The model, derived from an exclusively-developed computer program that has been approved and published by CRAN (4), yields unprecedented results concerning the teaching of three influential religions in East Asia, namely Confucianism, Taoism, and Buddhism.

The three religions make a good case study because, despite their deep-rooted influence in Vietnam over centuries, the values they uphold such as benevolence, loyalty-fidelity, justice-righteousness, propriety, compassion, non-violence, and honesty, have not completely deterred the acts of lying and violence (5). Indeed findings remain inconclusive on the relationships between lying/cheating and religion (6-8) as well as between violence and religion $(3,9-11)$. The primary reason is, different cultures or social groups recognize and penalize different sets of moral values (12-16). In other words, while all religions stress the need to cultivate virtues such as loyalty, reciprocity, honesty, and moderation, how these virtues are practiced in reality are not universal across cultures. In this regard, the present study contributes to the wave of scholarship on non-WEIRD (Western, Educated, Industrialized, Rich, and Democratic) countries for shedding light on the little-known behavioral variability and contradictions in the folklore of a developing Asian country. Here, we find that, despite the universality of lies and violence across societies, their interactions with institutional religious teachings can generate a cultural variance in terms of outcome.

First, we encode the details of 307 Vietnamese folk stories into binary variables. For example, if the main character behaves according to the core values of Buddhism, "VB" equals 1; if this character lies, "Lie" equals 1; if he or she commits violent acts, "Viol" equals 1 . The details of the stories concerning Confucianism and Taoism are encoded similarly $(4,5)$. We are also interested in whether external intervention from either human ("Int1") or the supernatural ("Int2") might influence the story's outcome. 

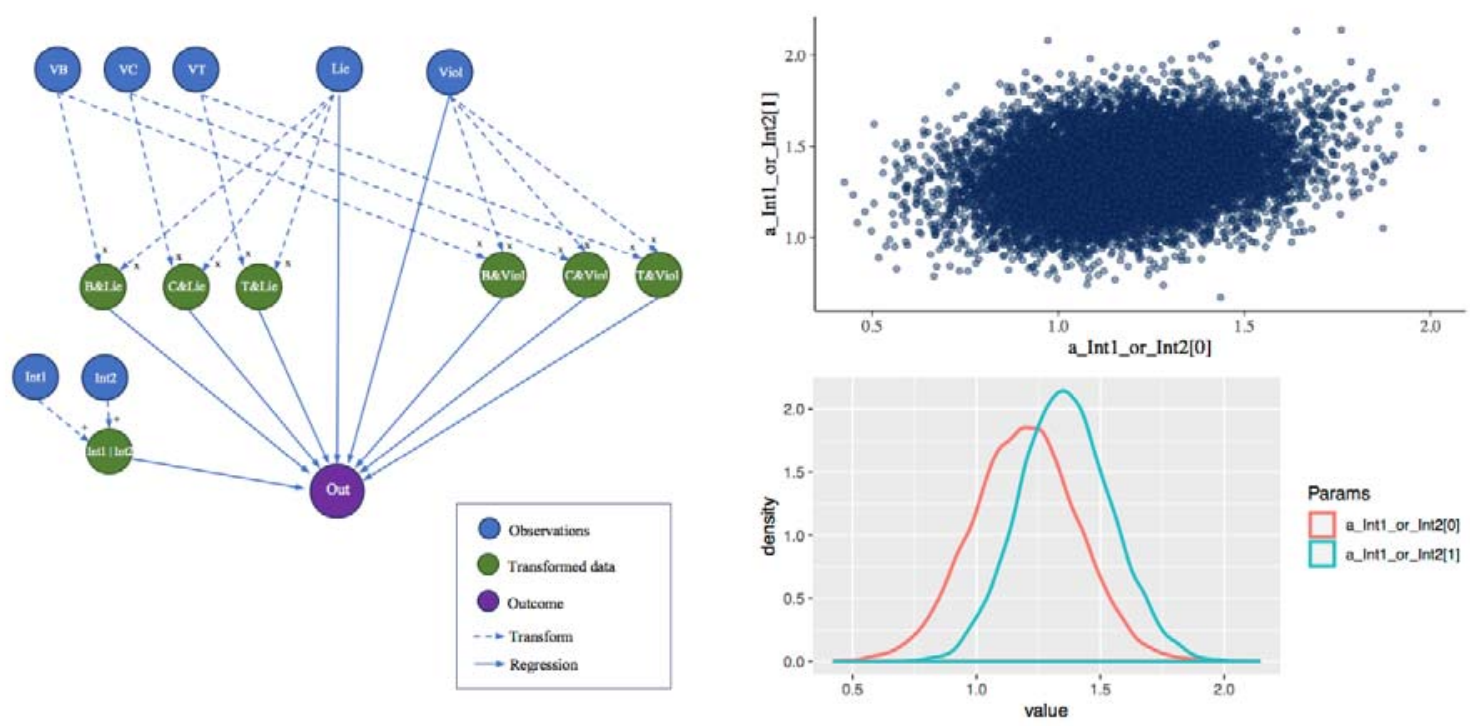

Figure 1. Conceptual model (Left) and the posterior probability distribution of the variable for intervention "Int1_or_Int2". (Upper Right) indicates pair values for the two states of Int1_or_Int2, and (Lower Right) shows the shifting of post-simulation distributions.

As we aim to understand the interactions among religious values and lies/violence, we combine these two categories of variables using mathematical operator (*), creating a new class of transformed data, which is coded by the green color in Figure 1. For instance, if a character behaves according to Taoist core values (wuwei, spontaneity, harmony, etc.) and still lies but does not act violently, the new transformed variables "T_and_Lie" and "T_and_Viol" will take on value 1 and 0 , respectively. We also combine "Int1" and "Int2" into "Int1_or_Int2," which stands for whether there is any external intervention or not. It is noteworthy that all variables except for "Int1_or_Int2" have the varying slope relationship with the variable outcome ("Out"), and that "Int1_or_Int2" has the varying intercept relationship with "Out".

We then perform the Bayesian MCMC analysis based on the hierarchical model presented in Figure 1 and measure the probability of an outcome of a character, given the religious values and the actions he or she commits. The Bayesian MCMC estimation uses our CRAN-published 'bayesvl' $\mathrm{R}$ package with Stan, for four Markov chains, each having 5,000 iterations, 2,000 warm-ups. The results indicate a good fit of the model with data. Specifically, the effective sample size is above 1,000 for all parameters of the model; the potential scale factor (Rhat) is approximately 1 for all parameters; all visual diagnostics show a good mixing and fast dying-out autocorrelation coefficients (4).

The right plane of Figure 1 presents the posterior distribution of the two values of the parameter intervention: 1 for intervention and 0 for no intervention. The charts show a similar pattern of the posterior distribution of the parameter, both distributed around mean values of 1.2 and 1.3, respectively, suggesting whether there are external interventions or not, there is an only negligible difference for the story outcome of the main characters. 

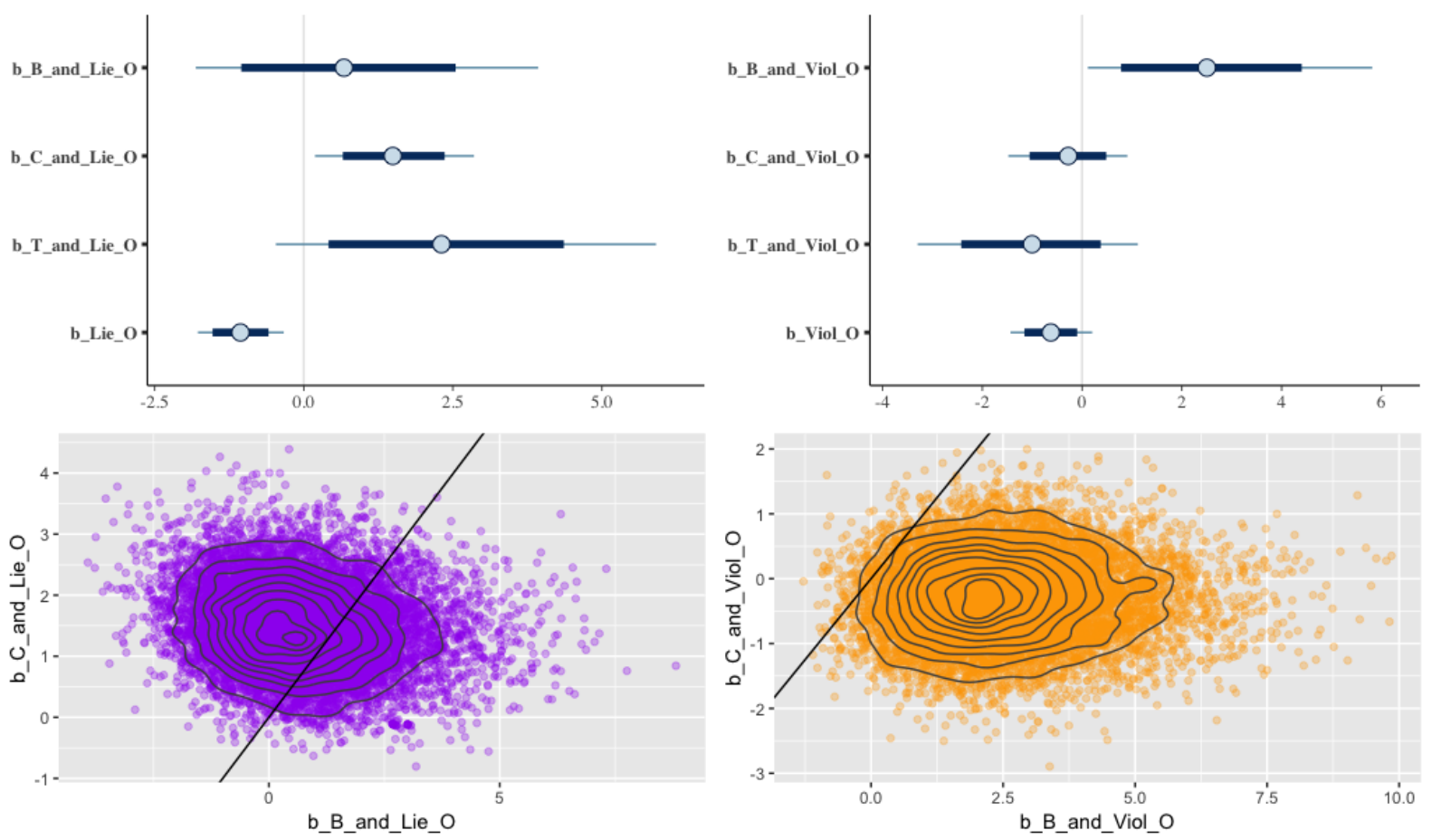

Figure 2. The MCMC posterior probability distribution of two sets of variables showing the interaction between lies, violence, and different religious values.

Figure 2 displays the probability density of parameters that involved the interaction between lies, violence, and different religious values. When we only consider the outcome of lying and violence alone, the results are intuitive. The value of coefficients b_Lie_O (the probability of a good outcome given the main character lies) and b_Viol_O (the probability of a good outcome given the main character commits violent acts) are negative (-1.05 and -0.62 , respectively). By contrast, when considering the interplay with religious values, it is not as straightforward to predict. The coefficients b_C_and_Viol_O and b_T_and_Viol_0 are negative $(-0.28$ and -0.96 , respectively $)$ but b_B_and_Viol_O is positive (2.55). This suggests the interaction among Confucian, Taoist values, and violent acts bring about bad outcomes for the main characters, whereas for the main characters whose actions represent Buddhist values, violence tends to bring a good outcome. For lying, interaction with all three teachings tends to bring about good outcome for the main character

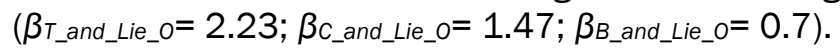

To make sense of the underlying mechanism behind these statistical patterns, we suggest examining which values in the Three Teachings are most observed by the laypeople. The tolerance for violence can be explained by the emphasis on karma in Buddhism - a concept might be loosely interpreted as "an eye for an eye," whereas the propensity for lying is attributable to the need to (i) preserve social order and being loyal and pious toward one's King and kins in Confucianism, and (ii) pursue spontaneity, and harmony with nature in Taoism. Historically speaking, Confucianism first started as a remedy to the chaotic, violent time of the Spring-Autumn Warring States, and Taoism was a response to the rigid way of life of the Confucianist, hence the retreat to nature and spontaneity. As these Chinese teachings prioritize social order, understandably they would be antithetical to violence and more acceptant to lying as the price in practice.

Given that all the stories were passed down through the oral tradition, they presumably do not evoke the true ideals of the three religions, but instead, reflect the psychology and understanding of the 
people at the time. Future studies could replicate the method beyond the folklore realm to see if these observations hold. What is highlighted here is a glaring double standard in the interpretation and practice of the three teachings: the very virtuous outcomes being preached, whether that be compassion and meditation in Buddhism, societal order in Confucianism, or natural harmony in Taoism, appear to accommodate two universal vices-violence in Buddhism and lying in the latter two. Attempts to make sense of contradictory human behaviors have pointed out the role of cognition in belief maintenance and motivated reasoning in discounting counterargument $(17,18)$. When it comes to religion, an individual's tolerance of contradictory religious teachings is not due to lower rationality standards but rather due to how such teachings fit the "inference machinery" in a plausible manner (19). This study takes a step further by showing how such action could even result in desirable outcomes, even if the findings may be limited to the folklore realm. What is troubling is the promotion of "the ends-justify-the-means" mentality when the acceptance of values counters to one's beliefs is correlated with positive outcomes.

\section{Acknowledgments:}

This research was supported by Al for Social Data Lab, Vuong \& Associates, and SDAG, Phenikaa University (Hanoi, Vietnam).

\section{Author contributions:}

Q.H.V. designed research; Q.H.V. and V.P.L. performed research; Q.H.V. and V.P.L. analyzed data; and Q.H.V. and H.K.T.N wrote the paper.

\section{Data deposition:}

The dataset is available from the Github (https://github.com/sshpa/bayesvl/tree/master/data), and CRAN 'bayesvl' R package (https://cran.r-project.org/package=bayesvl). The computer code is provided on https://github.com/sshpa/bayesvl/tree/master/examples (file: simulation_example.R) and graphs from https://github.com/sshpa/bayesvl/tree/master/LectureNotes/3.Graphs.

\section{References}

1. Graham J \& Haidt J (2010) Beyond beliefs: Religions bind individuals into moral communities. Personality and Social Psychology Review 14(1):140-150.

2. Gómez Á, et al. (2017) The devoted actor's will to fight and the spiritual dimension of human conflict. Nature Human Behaviour 1(9):673-679.

3. Purzycki BG \& Gibson K (2011) Religion and violence: an anthropological study on religious belief and violent behavior. Skeptic 16(2):22-27.

4. La VP \& Vuong QH (2019) bayesvl: Visually learning the graphical structure of Bayesian networks and performing MCMC with 'Stan.' The Comprehensive $R$ Archive Network. Available from: <https://cran.r-project.org/package=bayesvl>; v0.8.5 (May 24).

5. Vuong Q-H, et al. (2018) Cultural additivity: behavioural insights from the interaction of Confucianism, Buddhism, and Taoism in folktales. Palgrave Communications 4(1):143.

6. Bruggeman EL \& Hart KJ (1996) Cheating, lying, and moral reasoning by religious and secular high school students. The Journal of Educational Research 89(6):340-344.

7. Rettinger DA \& Jordan AE (2005) The relations among religion, motivation, and college cheating: A natural experiment. Ethics \& Behavior 15(2):107-129.

8. Mensah C \& Azila-Gbettor EM (2018) Religiosity and students' examination cheating: evidence from Ghana. International Journal of Educational Management 32(6):1156-1172. 
9. Atran S (2016) The devoted actor: Unconditional commitment and intractable conflict across cultures. Current Anthropology 57(S13):S192-S203.

10. Henrich J, Bauer M, Cassar A, Chytilová J, \& Purzycki BG (2019) War increases religiosity. Nature Human Behaviour 3(2):129-135.

11. Blogowska J, Lambert C, \& Saroglou V (2013) Religious prosociality and aggression: It's real. Journal for the Scientific Study of Religion 52(3):524-536.

12. McKay R \& Whitehouse H (2015) Religion and morality. Psychological Bulletin 141(2):447473.

13. Haidt J \& Graham J (2007) When morality opposes justice: Conservatives have moral intuitions that liberals may not recognize. Social Justice Research 20(1):98-116.

14. Haidt J \& Joseph C (2004) Intuitive ethics: How innately prepared intuitions generate culturally variable virtues. Daedalus 133(4):55-66.

15. Henrich J, Heine SJ, \& Norenzayan A (2010) The weirdest people in the world? Behavioral and Brain Sciences 33(2-3):61-83.

16. Graham J, Meindl P, Beall E, Johnson KM, \& Zhang L (2016) Cultural differences in moral judgment and behavior, across and within societies. Current Opinion in Psychology 8:125130.

17. Kaplan JT, Gimbel SI, \& Harris S (2016) Neural correlates of maintaining one's political beliefs in the face of counterevidence. Scientific Reports 6:39589.

18. Bersoff DM (1999) Why good people sometimes do bad things: Motivated reasoning and unethical behavior. Personality and Social Psychology Bulletin 25(1):28-39.

19. Boyer P (2001) Religion explained: The evolutionary origins of religious thought (Basic Books, New York). 